\title{
Typ-2-Diabetes-mellitus
}

\section{Therapieziele auch bei Nierenfunktionsstörung erreichen}

- Einen $\mathrm{HbA}_{1 \mathrm{c}}$-Wert von 6,5\% ohne Hypoglykämien und Gewichtszunahme zu erreichen, ist ein wesentliches Therapieziel bei Typ-2-Diabetes - auch für Patienten mit eingeschränkter Nierenfunktion.

Noch immer werden lange nicht bei allen $\mathrm{Pa}$ tienten die Therapieziele erreicht, beklagte Prof. Stephan Matthaei aus Quakenbrück. So lag in einem Register 2009 der $\mathrm{HbA}_{1 \mathrm{c}}$-Wert immer noch bei $40 \%$ der erfassten Patienten nicht $<7 \%$ [1]. Matthaei betonte, das Ziel muss weiterhin sein, bei mehr Patienten mit Typ-2-Diabetes den $\mathrm{HbA}_{1 c}$-Zielbereich $\mathrm{zu}$ erreichen als bisher, ihn dort zu halten und dabei Nebenwirkungen, insbesondere Hypoglykämien und eine ausgeprägte Gewichtszunahme, zu vermeiden - auch bei Patienten mit Nierenfunktionsstörungen. Als erstes Gliptin wurde Saxagliptin (Onglyza ${ }^{\circledast}$ ) speziell für diesen Indikationsbereich zugelassen.

Basis war eine Studie mit 170 Patienten mit einem schlecht kontrollierten Blutzucker $\left(\mathrm{HbA}_{1 \mathrm{c}}\right.$ zwischen 7 und $11 \%$ ) und einer Kreatininclearance von $<50$ $\mathrm{ml} / \mathrm{min}$, wie Prof. Baptist Gallwitz aus Tübingen erläuterte [2]. Unter Weiterführen der bisherigen Therapie erhielt randomisiert die Hälfte der Patienten einmal täglich 2,5 mg Saxagliptin, die andere Hälfte Placebo. Im Saxagliptinarm sank in den ersten 12 Wochen der $\mathrm{HbA}_{1 \mathrm{c}}$-Wert signifikant stärker als im Placeboarm ( $-0,86$ vs. $-0,44 \%, p=0,007)$, wobei Patienten mit einer moderaten Schwere der Nierenfunktionsstörung über die gesamte Beobachtungszeit besonders profitierten (Abb. 1). Die Sicherheitsdaten

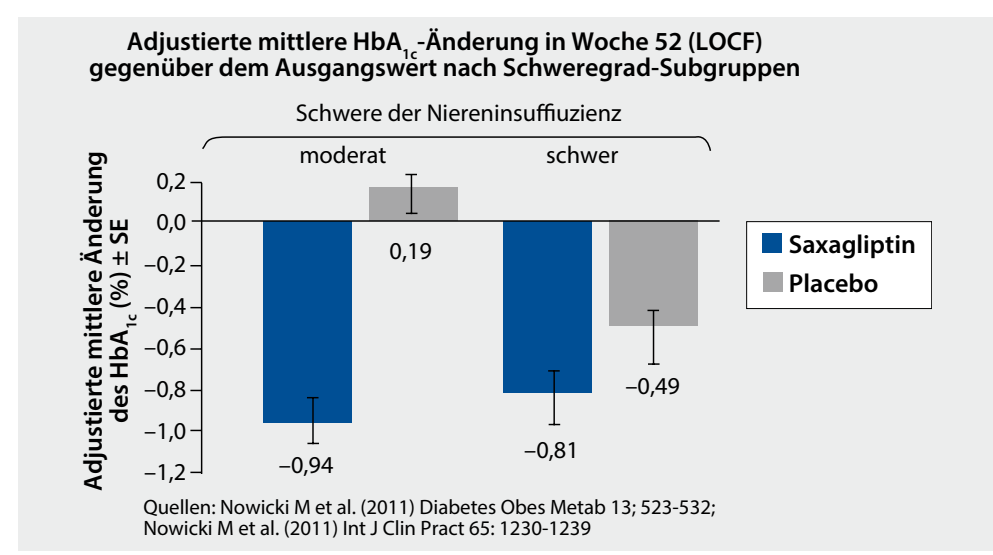

Abb.1: Nach 52 Wochen haben Patienten mit moderater Nierenfunktionsstörung hinsichtlich des $\mathrm{HbA}_{1 \mathrm{c}}$ besonders profitiert.

zeigten keine wesentlichen spezifischen Signale, so Gallwitz. Auch die Hypoglykämien waren bei der mit dem Gliptin intensivierten Therapie nicht wesentlich häufiger als im Placeboarm (4,7 vs. 3,5\%). Dabei blieb die Therapie mit Saxaglitpin auch über 52 Wochen gewichtsneutral. Wichtig sei aber, die Saxagliptindosis entsprechend der Niereninsuffizienz anzupassen, so Gallwitz: ( $5 \mathrm{mg}$ bei Kreatininclearance von $>50$ bis $\leq 80 \mathrm{ml} / \mathrm{min}, 2,5 \mathrm{mg}$ bei $\leq 50 \mathrm{ml} / \mathrm{min})$. $f k$

Quelle: Satellitensymposium beim 118. Kongress der Deutschen Gesellschaft für Innere Medizin e.V., Wiesbaden, 16.04.2012. Veranstalter: Bristol Myers Squibb
1. Schütt $\mathrm{M}$ et al. (2010) Exp Clin Endocrinol Diabetes 118: 490-5.

2. Nowicki $M$ et al. (2011) Diabetes Obes Metab 13; 523-532; Nowicki M et al. (2011) Int J Clin Pract 65: 1230-9.

\section{Neue Generation der Blutzuckermesssysteme Hohe Messgenauigkeit schützt vor Unterzuckerungen}

- „Moderne Diabetes-Therapien sind flexibel und richten sich nach dem Leben des Betroffenen und nicht umgekehrt", sagt Dr. Andreas Liebl, Chefarzt am Diabetes- und Stoffwechselzentrum der Fachklinik Bad Heilbronn. Regelmäßige Blutzuckerselbstkontrollen mit entsprechender Dokumentation sind dafür unverzichtbar.

Dies erfordert Blutzucker-Messsysteme, die einfach zu bedienen sind und eine hohe Messgenauigkeit garantieren, v.a. auch, um Hypoglykämien zu verhindern, so Liebl. Ebenso wichtig ist die Auswertung mit einer modernen Daten-Management-Software, die Trends aufzeigt und bei der Problemerkennung hilft. Denn „handschriftliche geführte Tagebücher sind nicht ausreichend“, sagte Liebl. Sie sind oft unleserlich, unvollständig, oder werden vom Patienten gar "geschönt“.

Ein Fortschritt ist das neue Blutzuckermessgerät Contour $^{\circledast}$ Next USB. Das Gerät zeichnet sich durch hohe Messgenauigkeit aus und übertrifft diesbezüglich die strengeren Kriterien der kommenden ISONorm.

Darüber hinaus ist es selbst von ungeschulten Patienten leicht und problemlos zu bedienen. Neben den Blutzuckerwerten können Insulin- und Kohlenhydratmengen eingegeben werden, sodass ein vollständiges Tagebuch geführt werden kann.

Der Arzt kann die Messwerte einfach nachvollziehen. das Gerät enthält neben einer integrierten Diabetes-Management-Software (Glucofacts ${ }^{\circledR}$ Deluxe) auch eine USB-Schnittstelle zur Übertragung der Werte auf den Computer. Dies ermöglicht langfristige Auswertungen und Dokumentationen.

sti

Quelle: Pressegespräch: Diabetes managen: Präzision im Fokus im Rahmen der 47. Jahrestagung der Deutschen Diabetesgesellschaft (DDG), Filderstadt, Mai 2012, Veranstalter: Bayer HealthCare. 